\title{
Prevalence of Dental Myth and Misconceptions among the Rural Population of Mangaluru City: A Cross Sectional Study
}

\author{
Renu M, Sabhya J, Shameen H, Sindhu P, \\ Vaishnavi D*, Anjana AP and Suhairath K \\ Department of Public Health Dentistry, AJ Institute of \\ Dental Science, Mangaluru, Karnataka, India \\ *Corresponding author: Vaishnavi D, Department of \\ Public Health Dentistry, AJ Institute of Dental Sciences, \\ Karnataka, India
}

Received: October 11, 2018; Accepted: November 21, 2018; Published: November 28, 2018

\begin{abstract}
Introduction: It is important to know about the myths especially in India where general and oral health is embroiled in various myths.

Objectives: The purpose of this study is aimed at assessing the prevalence of dental myths regarding oral health, among the rural community of Mangaluru.

Materials and Methods: A cross sectional study was conducted in a rural area of Mangaluru. A total of 200 individuals was included, data were collected using a pretested and validated sixteen term questionnaire, including demographic data, questions regarding dental myth. Data obtained were statistically analyzed using descriptive statistics. Chi-square test is the statistical analysis used.
\end{abstract}

Results: Almost all the participants believed in one or more dental myth. Majority of the participants, $71.5 \%$ believed that oral cancer is not commonly found in older age group. About $75 \%$ of the participants felt that there was no necessity to consult a dentist in the absence of pain. Around $55.5 \%$ believed that extraction of the teeth of upper jaw causes loss of vision. There was an equally contradicting notion about bleeding gums while brushing.

Conclusion: Various dental myth still lurk in the minds of the population, to discourage the unhealthy practices we the health professionals have to provide intensive health education and promote the adoption of healthy practices. It would be prudent to familiarize professionals to understand these myths and beliefs as they act as barriers towards seeking treatment.

Keywords: Beliefs; Gender; Dental Myths; Oral Health; Age

\section{Introduction}

Health care has become one of India's largest sectors. It is growing at a tremendous pace due to contribution from private and government groups. For any country to progress health care is the top most priority. Even though there are advancements in health care sector, various myths are inhibited in minds of people.

The word 'myth' comes from the Greek word "MYTHOS" which means the stories shared by a group of people which are a part of cultural identity, having a strong influence in seeking treatment during illness [1]. In scientific terms they are extensive and unquestioned false perspective [1]. Socio-cultural factors, false traditional beliefs, lack of proper education, non-scientific knowledge are responsible for seeding myths in the mind of people.

In the medical field too there are various beliefs among the population due to the failure of Indian education to inculcate rational thinking. Hence myths like this become more prevalent, for example; people should drink at least 8 glasses of water a day, we use $10 \%$ of our brains, hair and finger nails continue to grow after death; shaving hair causes it to grow back faster, darker, or coarser; reading in dim light ruins eye sight [9].
In India dental myths are very common. These myths can at times be detrimental and cause various degree of disabilities, hence have to be tackled at bud stage [2].

Oral disease being fourth most expensive disease to treat has caused global burden in underprivileged groups of developing and developed countries [8]. The major contributory factors are sociocultural and traditional beliefs, stress, anxiety, and illiteracy [5]. These myths are passed on from one generation to another generation thus causing deep rooted emotion. People often go to quacks when symptoms arise rather than to the dentist enhancing false beliefs. Various myths have been present since the past such as: Tooth fairy is a character in modern western culture is said to give a small gift in exchange for a deciduous tooth when it comes out of child's mouth. Years ago in Europe, children throw their lost tooth in mouse holes hoping that they would grow sharp teeth. In northern Europe, there was a tradition of tooth fee which was to be paid when a child lost his or her first tooth [2]. Children in Asian countries such as India, China, Japan, Korea and Vietnam toss their lower teeth on the roof and their upper teeth in ground, trusting that new teeth will grow towards the old teeth and be straight [2].

Poor oral health has inflicted intimidations, hampered social
Austin J Dent - Volume 5 Issue 6 - 2018

ISSN : 2381-9189 | www.austinpublishinggroup.com

Vaishnavi et al. (@) All rights are reserved
Citation: Renu M, Sabhya J, Shameen H, Sindhu P, Vaishnavi D, Anjana AP, et al. Prevalence of Dental Myth and Misconceptions among the Rural Population of Mangaluru City: A Cross Sectional Study. Austin J Dent. 2018; 5(6): 1120 
relation, lead to chronic diseases and has brought upon high financial cost. Hence adequate measures have to be taken to educate people regarding dental care.

The purpose of this study is to assess the prevalence of dental myths among the lower economic groups of Mangaluru district between the age groups of $20-60 \mathrm{yrs}$.

\section{Methodology}

A cross sectional questionnaire study was conducted in a rural area of Mangaluru, India. The houses that were locked were not considered as a part of the study. The study protocol was approved by the Institutional Ethical Committee (IEC).

The study subjects were selected based on convenience sampling. The following inclusion and exclusion criteria's were considered for the study.

\section{Inclusion criteria}

1) Study subjects gave verbal consent to be a part of the study.

2) Study subjects belonging to the age group of 20-60 years.

3) Study subjects who could comprehend the local language.

\section{Exclusion criteria}

1) Study subjects who are ill.

2) Study subjects who could not comprehend the questionnaire.

A 16 item structured, closed ended questionnaire was developed for the study (Table 1) [1]. There was no skip pattern and all the questions were to be answered. The questions 1-5 were based on myth regarding dental caries, the questions 6-9 were about myths regarding tobacco and oral cancer, the questions 10-16 were related to dental problem and treatment related dental myth. The data was collected for a period of 2 months.

The questionnaire was coded to maintain confidentiality of the data.

The data was fed into SPSS version 17 (SPSS Inc., Chicago, Ill, USA). Descriptive analysis of the data was done.

\section{Results}

A total of 150 houses were covered and 200 study subjects were included in the study. Among the 200 study subjects between the ages of 20-60 years who participated in the study $29.5 \%$, were males and $70.5 \%$ were females. The study subjects considered were from rural community of Mangalore. Since it was a door to door study the number of female participants were more as majority of them were homemakers and the number of male participants was less as they were out working.

About $61.5 \%$ of the participants believed that decay in the milk teeth needs to be treated while $55.5 \%$ of the participants had a misconception that placing cloves on the carious tooth relieved pain, Almost $49.5 \%$ of participants informed that they will opt for extraction of teeth than saving it.

Majority of the participants, $71.5 \%$ believed that oral cancer is not commonly found in older age group.

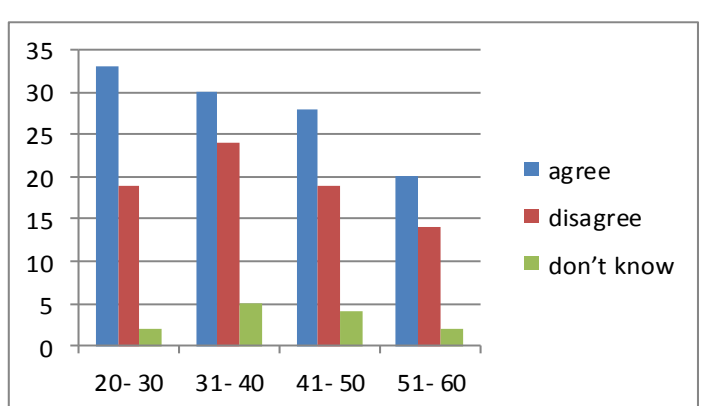

Figure 1: Comparison of prevalence of dental myth "all dental treatment are painful" among different age groups: (a) horizontal axis depicts age groups (b) vertical axis depicts mean value.

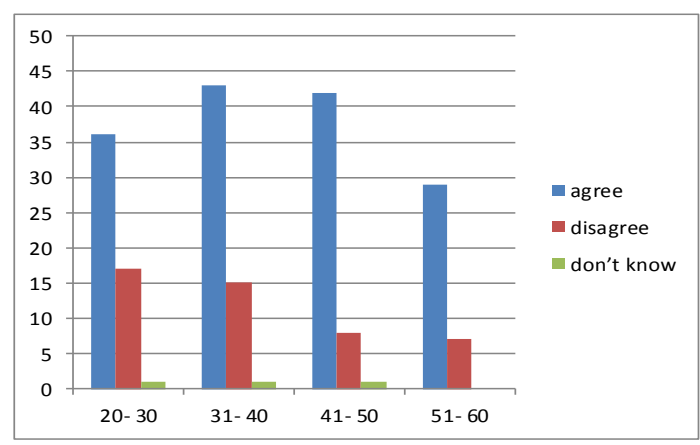

Figure 2: Comparison of prevalence of dental myth "if I am not in pain I do not need to visit the dentist" among different age groups: (a) horizontal axis depicts age groups (b) vertical axis depicts mean value.

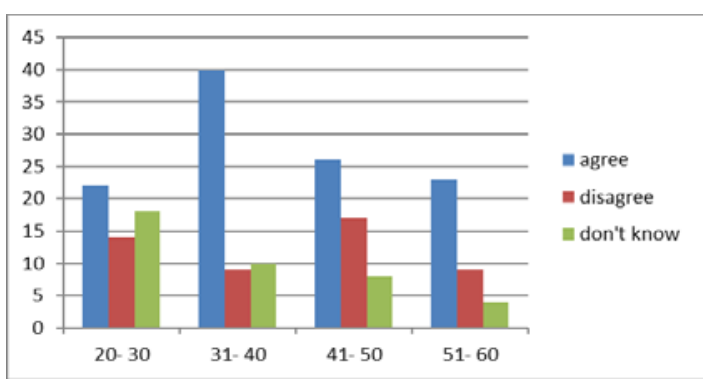

Figure 3: Comparison of prevalence of dental myth "extraction of teeth of upper jaw causes loss of vision" among different age group (a) horizontal axis depicts age group (b) vertical axis depicts mean value.

More than half of the study subjects, $55.5 \%$ agreed that all dental treatments were painful, while $75 \%$ of the participants felt that there was no necessity to consult a dentist in the absence of pain. Around $55.5 \%$ believed that extraction of the teeth of upper jaw causes loss of vision, while $48.5 \%$ of participants disagreed that scaling resulted in loose teeth.

There was an equally contradicting notion about bleeding gums while brushing. Almost $42.5 \%$ considered it normal where as $42 \%$ believed otherwise. Around $68.5 \%$ of them believed that usage of hard bristled brushes does not whiten the teeth.

\section{Discussion}

The deep seated irrational thoughts and beliefs among the masses 
Table 1: Responses of the Participants Regarding Dental Myth.

\begin{tabular}{|c|c|c|c|}
\hline Questions & Agree & Disagree & Don't Know \\
\hline 1. Dental decay occurs because my teeth are soft & $22.50 \%$ & $61 \%$ & $16.50 \%$ \\
\hline 2. Decay in the milk teeth requires no treatment as they are going to fall anyways & $35.50 \%$ & $61.50 \%$ & $3 \%$ \\
\hline 3. Placing cloves in the decayed tooth always relieves pain & $55.50 \%$ & $36.50 \%$ & $8 \%$ \\
\hline 4. Any tooth pain due to decay, it is better to extract rather than saving & $49.50 \%$ & $45.50 \%$ & $5 \%$ \\
\hline 5. Swelling caused by painful teeth should be fomented with hot water & $77 \%$ & $12.50 \%$ & $10.50 \%$ \\
\hline 6. Chewing of pan is good for oral health & $8 \%$ & $86.50 \%$ & $5.50 \%$ \\
\hline 7. Only aged people get cancer in mouth & $17.50 \%$ & $71.50 \%$ & $11 \%$ \\
\hline 8. Chewing of pan improves bowel movement after heavy meal & $34 \%$ & $35 \%$ & $31 \%$ \\
\hline 9. Betel nut quid chewing with slaked lime can keep gum problems away & $38 \%$ & $41 \%$ & $21 \%$ \\
\hline 10. All dental treatments are painful (Figure 1) & $55.50 \%$ & $38 \%$ & $6.50 \%$ \\
\hline 11. If I am not in pain I do not need to visit the dentist (Figure 2) & $75 \%$ & $23.50 \%$ & $1.50 \%$ \\
\hline 12. Extractions of teeth of upper jaw causes loss of vision (Figure 3) & $55.50 \%$ & $24.50 \%$ & $20 \%$ \\
\hline 13. A tooth once treated does not require any more treatment & $41.50 \%$ & $53 \%$ & $5.50 \%$ \\
\hline 14. Cleaning of teeth by dentist causes loosening of teeth & $21 \%$ & $48.50 \%$ & $30.50 \%$ \\
\hline 15. Bleeding of gums is normal during brushing & $42.50 \%$ & $42 \%$ & $15.50 \%$ \\
\hline 16. More you brush using hard bristles whiter your teeth becomes & $25 \%$ & $68.50 \%$ & $6.50 \%$ \\
\hline
\end{tabular}

of rural community, makes them less privileged and unfortunate to appreciate the advancements in dental profession.

According to the present study, $35.5 \%$ of study subjects believed that, decayed milk teeth required no treatment, as they will eventually exfoliate. Whereas, in studies conducted by Sharma R in Bengaluru, Karnataka and Yadav P in Jaipur, Rajasthan the prevalence of this myth among their study subject was higher $[1,3]$. This could be attributed to the amount of awareness being invoked through various dental health programs. Even though attempts are being made, a handful of people still believe that milk teeth requires no treatment.

Studies conducted in Bengaluru, Karnataka and Nagpur, Maharashtra showed that around $49.5 \%$ of the study participants believed in the analgesic action of cloves in decayed teeth [1,4]. It was found that almost $55.5 \%$ of the participants in the present study, most often resort to home remedies and 'treatment by quacks', rather than consulting a dental profession.

In the current study $49.5 \%$ of the individuals believed that extraction of teeth is better than saving it, which was higher than the study conducted by Mythri $\mathrm{H}$ [7]. This shows that people of the rural community are less aware of the existence of other treatment modalities to save a compromised teeth. The question of affordability and the number of appointments can be regarded as the possible limiting factor for opting such advanced care.

The role of print and digital media in creating awareness on the ill effects of tobacco should be highly appreciated. The prevalence of the myth that oral cancer is a disease of the aged, was very less among the rural population of Mangaluru. While only $17.5 \%$ agreed to this statement in our study, a similar study conducted in Navi Mumbai, Maharashtra by Singh S showed 100\% prevalence of this myth among their masses [8].

In the present study almost $55.5 \%$ believed that all dental treatments were painful whereas the same percentage of study subjects also believed that extraction of maxillary teeth leads to loss of vision. This was in accordance with the study conducted by Yadav $\mathrm{P}$ and much higher than the study conducted by Mythri H respectively $[6,7]$. This could be the result of a bad experience which is passed on to generations and in the times when the field of dentistry was still developing.

About $75 \%$ of the study subjects believed that it was not necessary to visit dentist if not in pain which is similar to the study conducted by Sharma R [1]. This high prevalence could be due to the notion that the tooth pain is an indicator of oral hygiene status. It is widely believed that if an individual is not in pain then his/her teeth are healthy.

In the present study $21 \%$ of the study subjects believed that cleaning of teeth causes it's loosening which was much lower than the alarming rate of $59.3 \%$ obtained from the study conducted by Sharma $\mathrm{R}$ [1]. This positive attitude could be due to awareness about dental procedures through awareness programs held at camps in schools, colleges and rural areas.

In the current study $42.5 \%$, study subjects believed that bleeding of gums was normal during brushing which is higher than the study conducted by Raina SA [4]. A hand full number of participants believed that bleeding gums is an effective way of losing bad blood from the body, while $42 \%$ of participants believed bleeding gums were a result of underlying pathology.

According to the present study $68.5 \%$ of the study subjects disagreed that brushing with hard bristles whitens their teeth which was much lower than the study conducted by Sharma R [1]. A small number of population believe that using hard bristle can damage the tooth and gums but some of them believe use of hard bristle is an effective method of tooth whitening. Hence adequate awareness must be created among people. 


\section{Limitations of the Study}

Data reported in this study cannot be generalized to the entire Indian population since the study was conducted in a rural setting. Exploration of research, to know other myths prevailing in the society is necessary. Also the probability with which the subject honestly handled the myths, hygiene and habit related issues are questionable. Further qualitative research studies including a large sample size is essential to circumvent the limitation of current study and more research is needed to validate the results of the study.

\section{Conclusion}

The results of current study states that even though there are several measures to educate the patients regarding health problem, myth still prevail among the common people. The best way to overcome it is to conduct a targeted programme to spread scientific dental practices. Introducing basic principles and concepts regarding oral hygiene of teeth as a part of education campaign to primary classes can curb these myths at grass root level.

\section{References}

1. Sharma R, Mallaiah P, Margabandhu S, Umashankar GK, Verma S. Dental myth, fallacies and misconception and its association with socio-dental impact locus of control scale. Int J. 2015; 1: 15.
2. Kiran GB, Pachava S, Sanikommu S, Simha BV, Srinivas R, Rao VN Evaluation of dent-o-myths among adult population living in a rural region of Andhra Pradesh, India: A cross-sectional study. Journal of Dr. NTR University of health sciences. 2016; 5: 130-136.

3. Yadav P, Shavi GR, Agarwal M, Choudhary P, Singh D. Myths and misconceptions about dentistry. A cross-sectional study. Arch of dent and med res. 2015; 1: 14-18.

4. Raina SA, Jain PS, Warhadpande MM. Myths and taboos in dentistry. International Journal of Research in Medical Sciences. 2017; 5: 1936-1942.

5. Singh SV, Tripathi A, Akbar Z, Chandra S, Tripathi A. Prevalence of dental myths, oral hygiene methods and tobacco habits in an ageing north Indian rural population. Gerodontology. 2012; 29: 53-56.

6. Khan SA, Dawani N, Bilal S. Perception and myths regarding oral health care amongst strata of low socio economic community in Karachi, Pakistan. J Pak Med Assoc. 2012; 62: 1198-1203.

7. Mythri Kumar RS. Perceived myths about oral health in India. Indian J Dent Res. 2015; 26: 333.

8. Singh S, Sirodhar G, Jain R, Dupare R. Myths related to Dental Decay and Tobacco Consumption: Debunked. International journal of preventive and clinical dental research, January-March. 2016; 3: 9-14.

9. Mamdani b. Medical myths. Indian Journal of Medical Ethics. 2008.
Austin J Dent - Volume 5 Issue 6 - 2018

ISSN : 2381-9189 | www.austinpublishinggroup.com

Vaishnavi et al. (๑) All rights are reserved
Citation: Renu M, Sabhya J, Shameen H, Sindhu P, Vaishnavi D, Anjana AP, et al. Prevalence of Dental Myth and Misconceptions among the Rural Population of Mangaluru City: A Cross Sectional Study. Austin J Dent. 2018; 5(6): 1120 